\title{
Eye Detection based on Viola \& Jones Detector, Skin Color, and Eye Template
}

\author{
Samir El Kaddouhi ${ }^{1}$, Abderrahim Saaidi ${ }^{1,2}$ and Mustapha Abarkan ${ }^{1}$ \\ ${ }^{1}$ LSI, Department of Mathematics, Physics and Computer Science \\ Polydisciplinary Faculty of Taza, Sidi Mohamed Ben Abdellah University \\ B.P. 1223, Taza, Morocco \\ ${ }^{2}$ LIIAN, Department of Mathematics and Computer Science, \\ Faculty of Sciences, Dhar-Mahraz, Sidi Mohamed Ben Abdellah University, B.P \\ 1796 Atlas- Fez, Morocco \\ samir.elkaddouhi@gmail.com,saaidi.abde@yahoo.fr, \\ mustapha.abarkan@usmba.ac.ma
}

\begin{abstract}
In this paper, we propose a new method of eye detection based on Viola \& Jones detector, skin color and eye template. Our method is processed into three steps: In the first step, faces are detected by using the Viola \& Jones detector. In the second step, face images are segmented into skin regions and non-skin regions with a threshold calculated by a combination of rules proposed on elements of the three color spaces RGB, $\mathrm{HSV}$ and YCbCr. The third step is the matching of an eye template with non-skin regions. Our method is tested on the FEI database available online, as well as a set of personal images and other from the Internet. The obtained results are satisfactory in terms of the quality of detection and rapidity.
\end{abstract}

Keywords: eye detection, face detection, skin color, Viola-Jones detector, eye template

\section{Introduction}

Eyes detection is a very interesting field of research that verifies the presence of eyes and locates their positions in an image. Similarly, it is often the first step in such applications such as face recognition [1,2], video surveillance [3,4], facial expression recognition [5,6], age estimation [7], gaze tracking systems [8,9], and driver fatigue monitoring systems [10-13].

However, eyes detection is a difficult task because of the computation time, the lighting conditions and the presence or absence of structural components such as glasses. To overcome these problems, various techniques have been developed in recent years and can be divided into four categories [14,15]: Template-matching methods, feature-based methods, appearance-based methods, and hybrid methods. In template-matching methods [16,17], an eye template is constructed and then compared with the different regions of the image to determine those that are the eyes. These methods are easy and quick, but they cannot be treated with eye variations in the scale, expression, rotation and lighting. The feature-based methods [18-20] explore the characteristics of the eye such as shape, intensity or gradient information. Although these methods are generally effective, they lack precision in the images with low illumination. In appearance-based methods [21-26], eyes are detected on the basis of their appearance photometric. These methods treat the detection of eye as a classification problem (eye/non-eye). The classification is performed by using a set

Received (November 27, 2017), Review Result (March 22, 2018), Accepted (April 1, 2018) 
of learning images and by using learning algorithms such as Neural Networks, AdaBoost algorithm, and Support Vector Machine (SVM). These algorithms require a large number of training data to enumerate all the possible eyes appearances. Hybrid methods [27-34] combine several methods to exploit their advantages and to avoid their disadvantages.

In this article, we present an efficient eye detection method. It is based on the Viola-Jones method and the skin color. Our technique involves three steps: in the first one, faces are detected using the Viola \& Jones detector. In the second step, face images are segmented into skin regions and non-skin regions with a threshold calculated by a combination of rules proposed on elements of the three color spaces RGB, HSV and YCbCr. In the third step, the eyes are detected by matching of eye template with non-skin regions.

The different steps of our eyes detection method are presented in Figure 1 below.

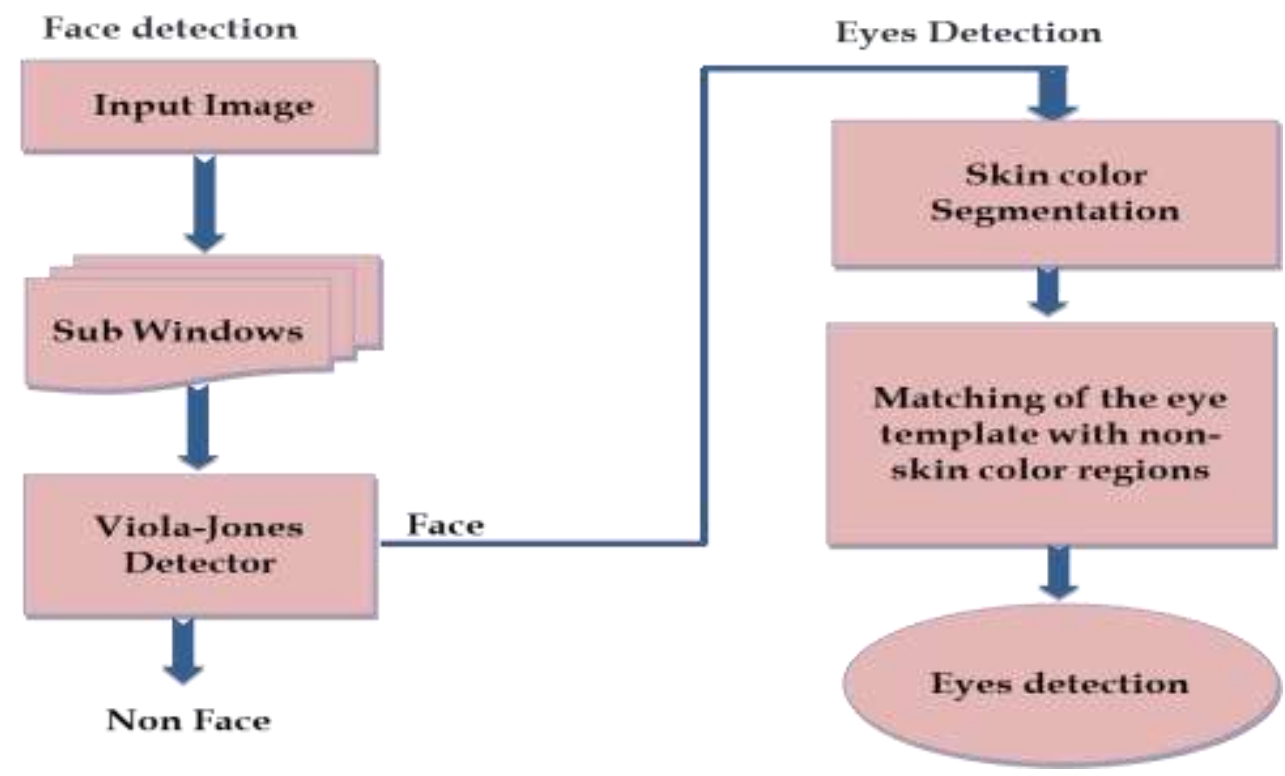

Figure 1. Diagram of Eyes Detection by our Method

The rest of this paper is organized as follows. In the second part, we provide a brief overview of the related word of the eyes detection. In the third and the fourth part, we dwell on our approach and its experimental results. The last part is devoted to the conclusion.

\section{Related Work}

In recent decades, several eye detection methods are proposed and can be classified into four categories: Template-matching methods, feature-based methods, appearance-based methods, and hybrid methods.

Template-matching methods [16,17]: Among the eye detection methods based on template matching, there is the method proposed by F. Rehab et al., [17] uses a deformable template (the template size and the rotation angle are adjusted to find the search area that best fits the reference template) and a matching algorithm based on the normalized cross-correlation coefficient (NCC) and particle swarm optimization (PSO).

Feature-based methods [18-20]: The method of eye detection presented by Jiatao Song et al., [18] starts with: Extracting the edge binary images from the grayscale 
image based on a multi-resolution wavelet transformation, extracting regions and eye segments from the binary image, and the location of the eye using light points and intensity information. Lilipta Kumar Bhatta, et al., [19] propose an approach to extract facial features (the eyes, the nose, and the mouth) which takes place in three steps. First, the faces are detected by the Sobel edge detector, and the intensity values of the edges. Then, in the detected faces, the skin regions are extracted in the $\mathrm{YCbCr}$ space. Then, the left eye is located in the left part of the skin color image considering that the point with minimum intensity value is as position of the left eye. The right eye is automatically on the same distance from the center due to the symmetry. Muhammad Affan Zia et al., [20] introduce an eye detection approach that takes place in two steps: the first step is to detect the faces in the image by segmenting the skin color in the Lab color space. The next step is to locate the eyes in the facial area by using the circular Hough transformation.

Appearance-based methods [21-26]: Many appearance-based approaches are proposed. For example, Riti Sharma et al. [22] develop an eye detection method that uses the histogram of oriented gradients (HOG) for reducing the size of the image and the SVM for classification. Vijayalaxmi et al., [23] perform the detection of eyes using a neural network and a Gabor filter. The proposed algorithm is divided into two phases: training and detection. In the training phase a set of images of the eyes and non-eyes are used to form the neural network, and in the detection phase, the eyes are detected using the formed network. Peng Wang et al., [25] present an eye detection method based on the AdaBoost algorithm, new rectangular features and geometric features. This article uses the rectangular features proposed by Viola-Jones [35] and adds other features to build a cascade classifier for eye detection. Then the symmetry characteristic of both eyes, and some geometric features are adopted to correct the detection errors obtained by the cascade classifier (the eyebrows, the mouth, the eyes big or small).

Hybrid methods [27-34] that combine between several techniques to exploit their advantages and avoid their disadvantages. More articles are cited: S. El Kaddouhi et al., [28], propose a new method for detecting faces and eyes. It is based on the skin color, face shape and corners points of Harris. In this method, faces are detected by the segmentation of the skin areas and by using the shape operations (surface, ratio, eccentricity). Then, the eyes are located by matching of an eye template with a small area determined by clustering of neighboring corners points. Mingxin YU et al., [29] present an eye detection technique based on the grayscale variance filter and the support vector machine. The variance filter is used to eliminate most images from non-eye regions to keep fewer eye candidate regions. Then the precise location of the regions of both eyes is determined by the SVM classifier. M. Hassaballah, et al., [31] suggest an eye detection method based on gray intensity variance and independent component analysis. First, the face area is located by the face detector boosted cascade. Then the top half of the face is divided into a large number of overlapping windows and applying a variance filter to select between these windows those candidate eyes. Then all the selected windows are checked using the independent component analysis to select only two windows that are the windows of the right and left eyes. The method of detection and localization of the eyes centers presented by Zhaocui Han, et al., [32] use the method of Viola and Jones to detect the faces in an acquired image. Then extract the area combining the eye and the neighboring eyebrow using the Haar features. Then locate the eyes by integrating the gradient distribution features, and curvelet features, and using the principal component analysis. 


\section{Our Method}

Our suggested method for eye detection contains three algorithms. The first algorithm involves using a simple and effective method for face detection which is based on the Viola \& Jones detector. In the second step, face images are segmented into skin regions and non-skin regions with a threshold calculated by a combination of rules proposed on elements of the three-color spaces RGB, HSV and YCbCr. In the third step, the eyes are detected by the matching of an eye template with the non-skin regions.

\subsection{Face Detection by Viola \& Jones Detector}

In the proposed method, the Viola-Jones detector [35] are chosen as a face detection algorithm, because of its high detection rate, and its ability to run in real time. This detector is comprised of three main concepts: Employing rectangular Haarlike features (see Figure 2 (a)) and a learning method based on Adaboost, (see Figure 2 (b)) and the attention cascade structure (see Figure 2 (c)).

In the Viola-Jones method there are two essential phases:

\section{- The Learning Phase:}

The learning is done on a very large set of positive (face) and negative (non-face) images. Many thousands of examples are usually needed. This learning includes:

- Calculation of Haar-like features on the positive and negative examples;

\section{- Construction of weak classifiers}

- Formation of the cascade: In each stage of the cascade, a strong classifier is formed by the combination of several weak classifiers.

\section{- The Detection Phase:}

The detection is applied to a test image, in which it is desired to detect the face. There are two steps:

1. scan the image with an initial window of size $24 \times 24$ pixels (then increase by a factor of 1.25), and application of the cascade to each sub-window, starting with the first stage of cascade:

- The calculation of the Haar-like features on the sub-window;

- The calculation of the response of the strong classifier of the current stage,

- If the answer is positive, passed at the following stage, else passed at the next sub window.

- Finally, the sub window is declared positive if all stages responds positively;

2. Merge overlapping multiple detections to return only one result. 


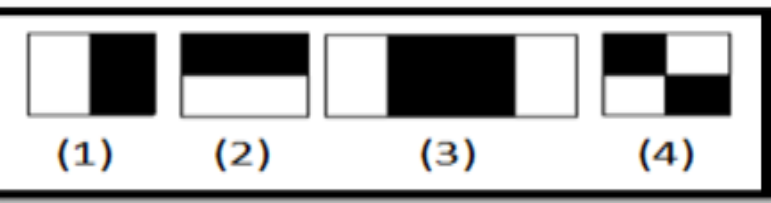

(a)

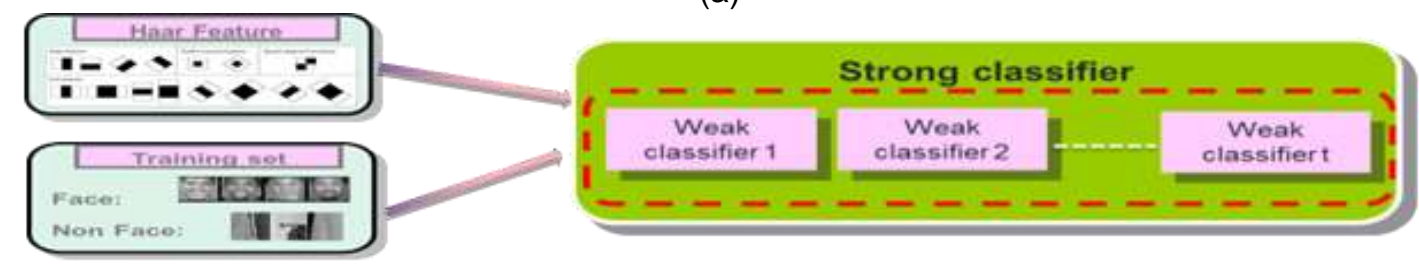

(b)

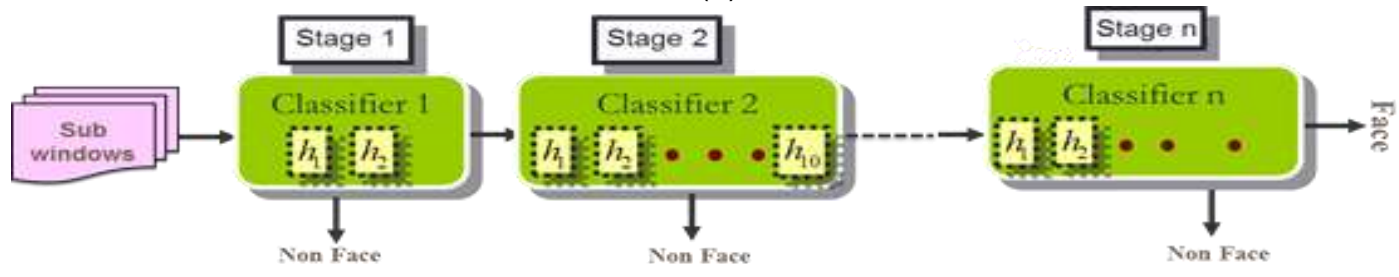

(c)

Figure 2. Components of the Viol-Jones Detector. (a): Examples of Haar like Features. (b): Training of Classifiers by AdaBoost Algorithm. (c): Cascade of Classifiers

Figure 3 illustrates some results of face detection in different situations.

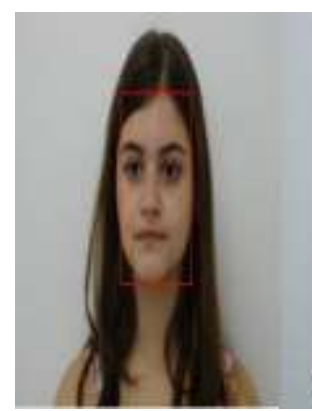

(a)

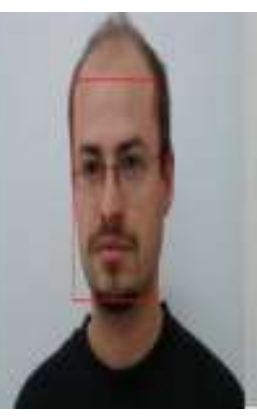

(b)

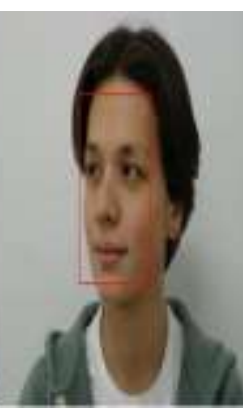

(c)

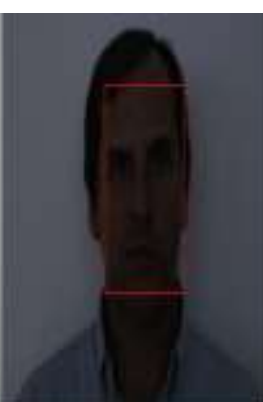

(d)

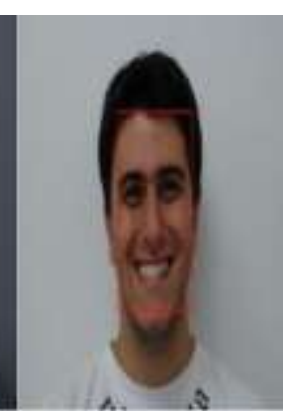

(e)

Figure 3. Face Detection Performed by our Method: Frontal Face (a), Frontal Face with Glasses (b), Profile Face (c), Image with Low Lighting (d), Face with Smile (e)

\subsection{Determination of Non-Skin Regions}

Generally, the human face contains skin regions and non-skin regions (eyes, mouth, beard, eyebrows). Thus, the detection of the non-skin regions in the faces make it possible to determine regions with a high probability of being eyes. Otherwise, to determine the non-skin areas, we follow the following steps:

\subsubsection{Choice of Color Space}

Several studies have shown that the variability of skin color was more than the difference in intensity rather than chrominance. However, the most suitable color spaces for segmenting skin regions are those separating luminance from chrominance [36]. Among these spaces we used rgb normalized, HSV and YCbCr. 


\subsubsection{Rules Defining the Skin Regions}

Several rules are used to determine regions with skin color, but the rules that give the best results are those used in [28] (see Table 1):

Table 1. Rules Defining the Skin Regions

\begin{tabular}{|l|l|l|}
\hline Space & \multicolumn{2}{|l|}{ Rules } \\
\hline \multirow{3}{*}{ Normalized rgb } & A & $0.45 \leq r \leq 0.7$ \\
\cline { 2 - 3 } & B & $0.23 \leq g \leq 0.55$ \\
\hline \multirow{2}{*}{ YCbCr } & C & $77 \leq C r \leq 127$ \\
\cline { 2 - 3 } & D & $133 \leq C b \leq 173$ \\
\hline \multirow{2}{*}{ HSV } & E & $0 \leq H \leq 0.2$ \\
\cline { 2 - 3 } & F & $0.2 \leq S \leq 0.7$ \\
\hline
\end{tabular}

\subsubsection{Face Image Segmentation}

The segmentation of images into skin and non-skin regions is done by using previously defined rules. It is performed using a threshold calculated by the combination of the rules proposed in Table 1. These rules are combined as follows:

$$
((A) A N D(B)) O R((C) A N D(D)) O R((E) A N D(F))
$$

Figure 4 shows segmentation of images into skin and non-skin regions.

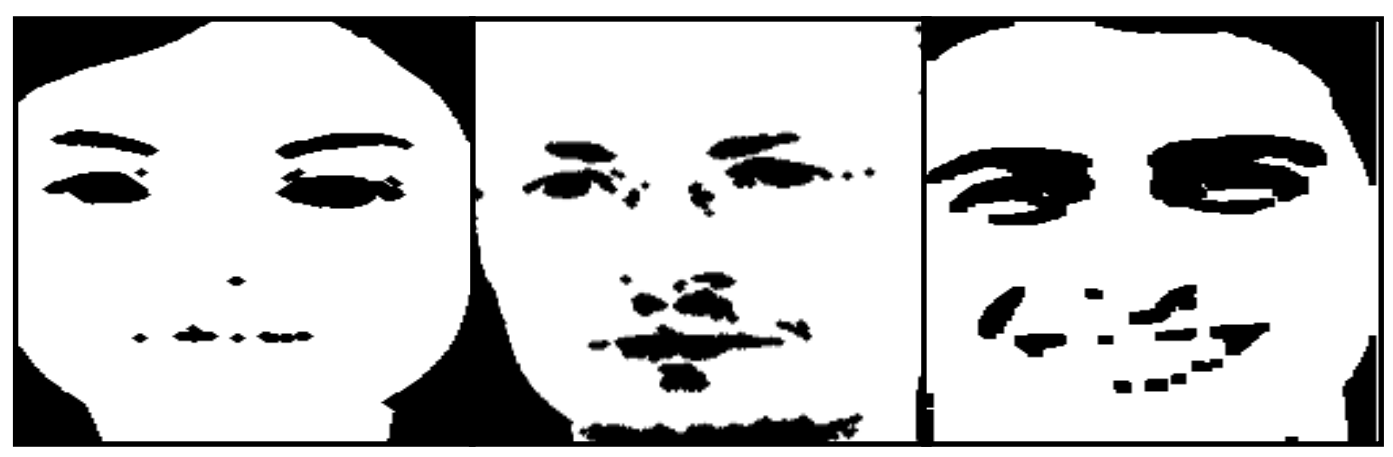

(a)

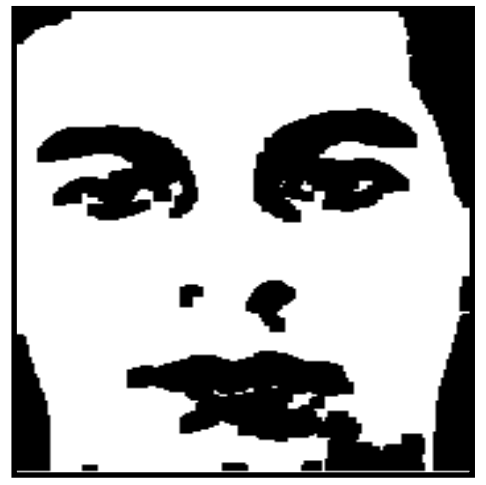

(b)

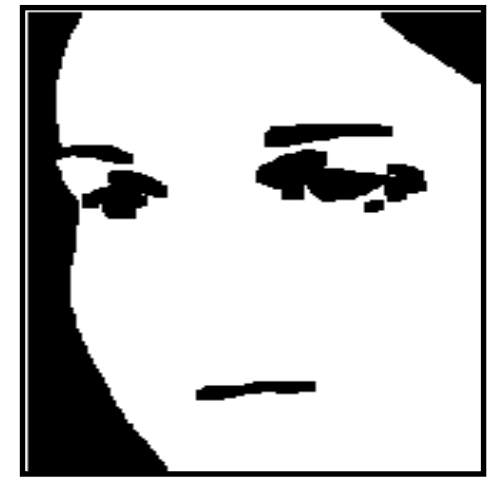

Figure 4. Segmentation of Face Images into Skin and Non-Skin Regions: Frontal Face (a), Frontal Face with Glasses (b), Face with Smile (c), Image with Low Lighting (d), Profile Face (e) 


\subsubsection{Determination of Non-Skin Regions}

In the image obtained after the segmentation, we see that there are two types of regions: white regions that represent the areas of the skin, and black regions that represent the nonskin areas. Each of non-skin areas is likely to be an eye. Thus, the location of these regions can determine areas likely to be eyes as shown in Figure 5 below.

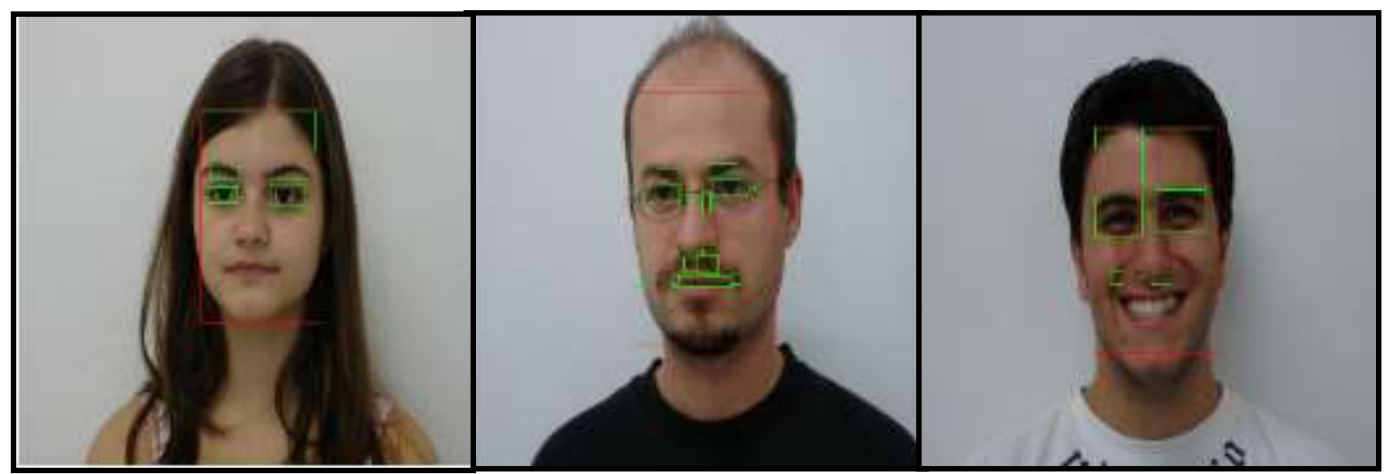

(a)

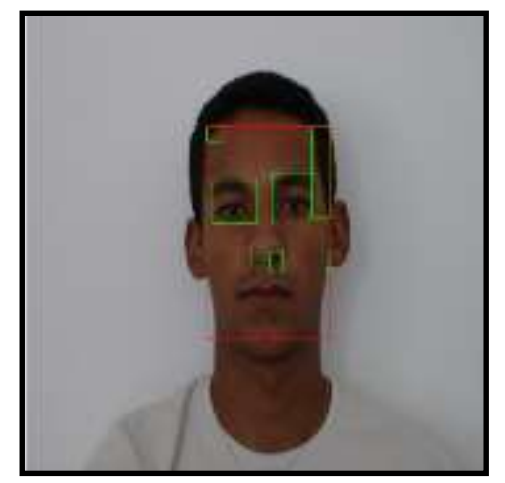

(d) (b)

(c)

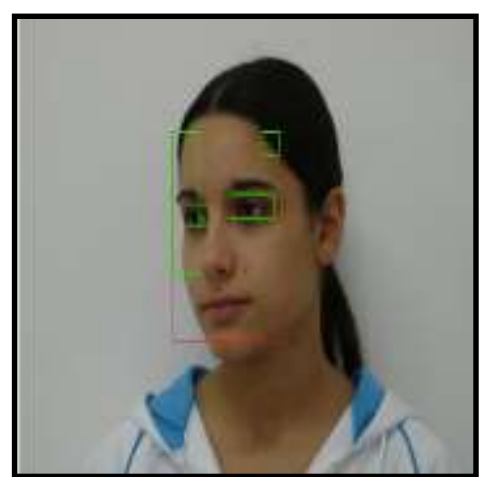

(e)

Figure 5. Determination of Non-Skin Regions: Frontal Face (a), Frontal Face with Glasses (b), Face with Smile (c), Image with Low Lighting (d), Profile Face (e)

\subsection{Eye Detection by Template Matching Method}

After getting the non-skin regions, an eye template $(\mathbf{T})$ is created by the average of a set of eyes images of several people taken from the FEI database. Then the correlation between this template and the different non-skin regions is calculated by using the normalized crosscorrelation function (NCC) defined by the formula 2 [17].

$$
\operatorname{NCC}(x, y)=\frac{\sum_{x, y} \delta_{I(x, y)} \delta_{T(x-u, y-v)}}{\left\{\sum_{x, y} \delta_{I(x, y)}{ }^{2} \sum_{x, y} \delta_{T(x-u, y-v)}\right\}^{0.5}}
$$

Where:

$$
\begin{aligned}
& u \in\{1,2,3, \ldots, p\}, \\
& v \in\{1,2,3, \ldots, q\} \\
& x \in\{1,2,3, \ldots, m-p+1\}, \\
& y \in\{1,2,3, \ldots, q-n+1\} \\
& \delta_{I(x, y)}=I(x, y)-\overline{I_{u, v}} \\
& \delta_{T(x-u, y-v)}=T(x-u, y-v)-\bar{T}
\end{aligned}
$$


$\overline{I_{u, v}}=\frac{1}{p q} \sum_{u v} I(x, y)$

$\bar{T}=\frac{1}{p q} \sum_{u v} T(x-u, y-v)$

I is the input image

$\mathrm{T}$ is a template

$\mathrm{m}$ and $\mathrm{n}$ are the sizes of image.

$p$ and $q$ are the sizes of template.

Figure 6 shows the detection of the eyes by our method. The red rectangles represent the detected face, and the green rectangles represent the detected eyes.

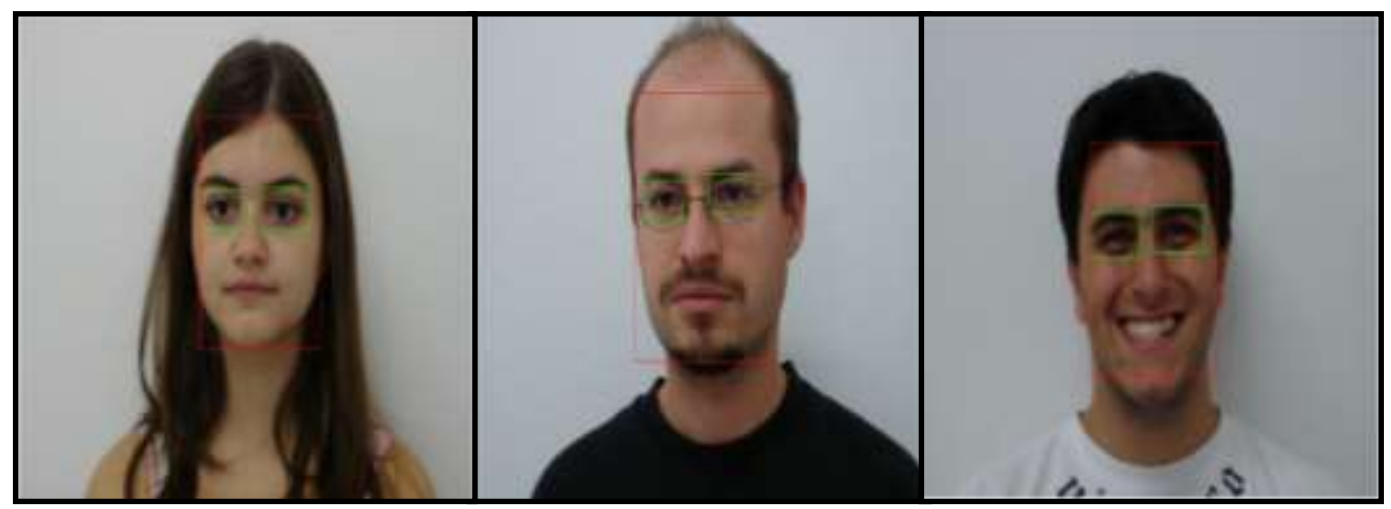

(a)

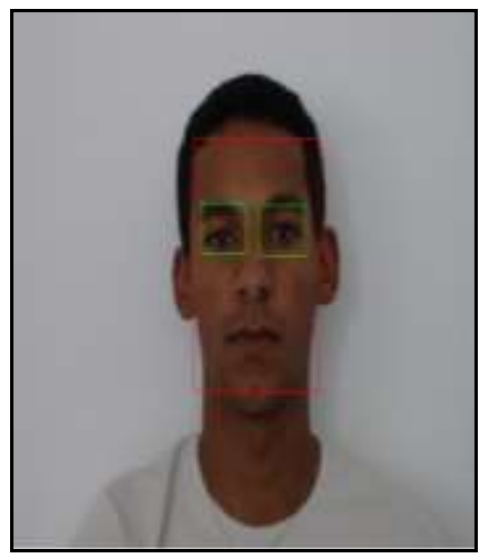

(d) (b)

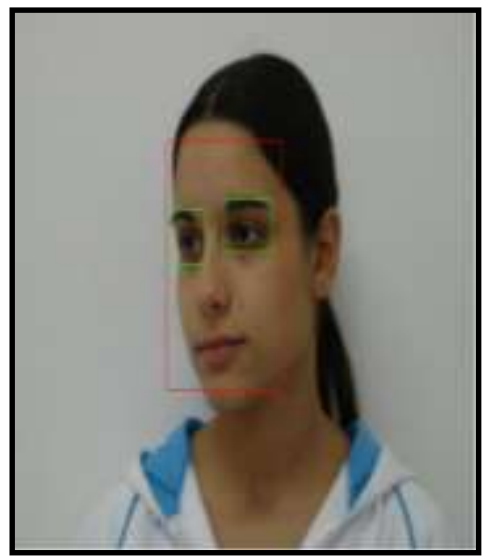

(e)

Figure 6. Face and Eye Detection by our Method: Frontal Face (a), Frontal Face with Glasses (b), Face with Smile (c), Image with Low Lighting (d), Profile Face (e)

We observe that the proposed approach allows to detect the eyes in the color images for the frontal faces (a), the faces with glasses (b), the faces with smile (c), image with low lighting (d), and the profiles faces (e).

\section{Experimental Results}

To evaluate the performance of our method and compare it with the other methods of eye detection, we used the FEI database [37], a set of additional personal pictures and other issues of Internet. The evaluation is based on two indicators: the correct 
detection rate (the number of correctly detected eyes over the total number of eyes), and the false detection rate (the number of false detected eyes over the number of detections).

\subsection{Simulation (Dataset)}

We tested our method on the FEI database [37] available online. It contains a set of images of 200 people taken in 14 different situations (lighting, pose ...). We tested our method in 2800 images; each image contains a single face. This test gives very important results (Table 2). Indeed, for all the images, we obtained a correct detection rate exceeds $96 \%$ and a false detection rate approximately $2 \%$.

Table 2. Eye Detection Results Obtained using our Approach

\begin{tabular}{l|c|c|c|c|c|c}
\multicolumn{2}{c|}{ Characteristics of the database FEI [37] } & \multicolumn{3}{c}{ Eye detection with our method } \\
\hline Images & $\begin{array}{c}\text { Number of } \\
\text { images }\end{array}$ & $\begin{array}{c}\text { Number of } \\
\text { eyes }\end{array}$ & $\begin{array}{c}\text { Number of correct } \\
\text { detection }\end{array}$ & \multicolumn{2}{c}{$\begin{array}{c}\text { Number of false } \\
\text { detection }\end{array}$} \\
\hline Frontal & 1400 & 2800 & 2761 & $98,60 \%$ & 19 & $0,68 \%$ \\
\hline Profile & 800 & 1171 & 1109 & $94,70 \%$ & 81 & $6,80 \%$ \\
\hline Dark lighting & 400 & 762 & 694 & $91,07 \%$ & 13 & $1,83 \%$ \\
\hline $\begin{array}{l}\text { facial expression } \\
\text { (smile) }\end{array}$ & 200 & 400 & 392 & $98,00 \%$ & 5 & $1,25 \%$ \\
\hline Occultation (glasses) & 66 & 115 & 109 & $94,78 \%$ & 8 & $6,83 \%$ \\
\hline \multicolumn{1}{c|}{ Total } & $\mathbf{2 8 0 0}$ & $\mathbf{5 1 3 3}$ & $\mathbf{4 9 5 6}$ & $\mathbf{9 6 , 5 5 \%}$ & $\mathbf{1 1 8}$ & $\mathbf{2 , 3 2 \%}$
\end{tabular}

For frontal faces under standard conditions of lighting, and in the absence of occlusions (glasses), our method achieved a correct detection rate exceeding $98 \%$ and a false detection rate less than $1 \%$. Our technique is able to detect the eyes in the images with a weak variation of lighting. But it fails when there is a strong variation of lighting, which justifies the decrease of the rate of true detection in the case of the dark images. For profile faces and in the presence of glasses, our approach achieved a satisfactory result. We obtained a correct detection rate more than $94 \%$, and a false detection rate less than $6 \%$. The presence of the facial expressions does not affect the quality of our approach, because we obtained a correct detection rate higher than $97 \%$, and a low false detection rate.

To illustrate the detection of the eyes in the presence of the various factors that disturb the detection process, we present in Figure 7 some example of results obtained. We see that our method can detect eyes with precision, even for profile faces (a1 and a2) faces with smile (b1 and b2), face with glasses ( 1 and c2), and dark images (d1 and $\mathrm{d} 2)$.

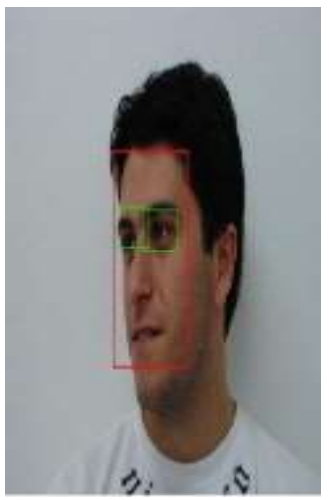

(a1)

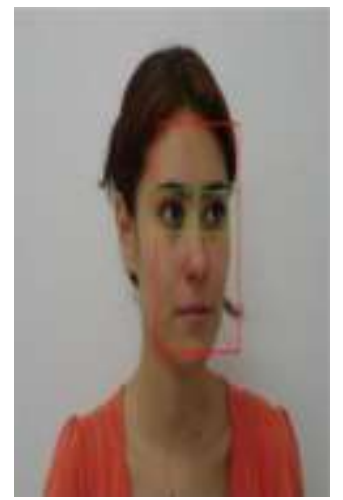

(a2)

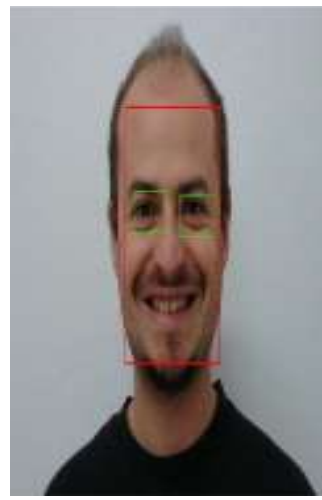

(b1)

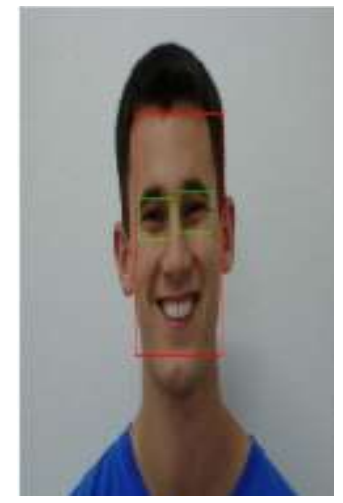

(b2) 


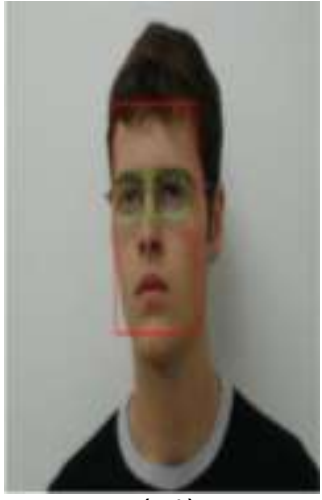

(c1)

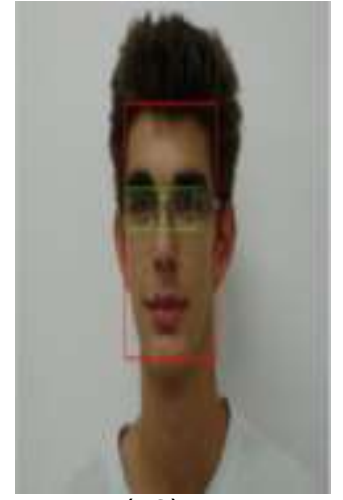

(c2)

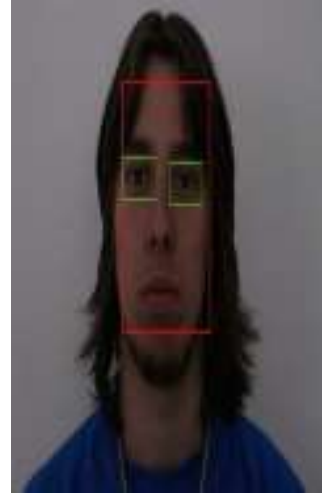

(d1)

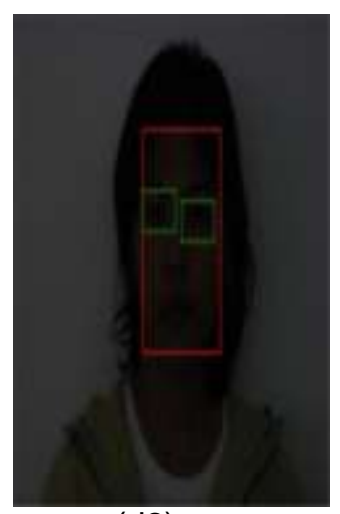

(d2)

Figure 7. Eye Detection in the Presence of Various Constraints : a1 and a2 : Profile Face. b1 and b2 : Face with Smile. c1 and c2 : Face with Glassses. d1 and d2 Dark Images

\subsection{Comparison}

To confirm and demonstrate the quality of our approach, we compared our results with those of three other eye detection methods recently published in the field and using the same database (FEI). The results obtained are shown in Table 3.

\section{Table 3. Results of Eyes Detection Obtained with our Method and Compared to Those in the Literature}

\begin{tabular}{cc}
\hline & correct detection rate \\
\hline Our method & $\mathbf{9 6 , 5 5 \%}$ \\
L.K.Bhatta et al. [19] & $96,5 \%$ \\
S.E.Kaddouhi et al. [28] & $95,86 \%$ \\
Mingxin YU et al. [29] & $95,2 \%$ \\
\hline
\end{tabular}

Table 3 shows the correct detection rate obtained with our method compared with that obtained with the other. We see that our method gives satisfactory results compared to other approaches. It gives a better rate than all other methods.

\subsection{Real Data}

To show the quality of our algorithm in the case of complex images, we tested our method on 50 images containing 336 eyes. These images are complex with body parts, several people and backgrounds can disrupt the process of detection. We obtained very important results (Table 4). We have detected 310 eyes and 31 false detections. We achieved a correct detection rate greater than $92 \%$, compared to the false detection rate remains low $(9.09 \%)$.

\section{Table 4. Eye Detection Results Obtained}

\begin{tabular}{c|c|c|c|c} 
Number total of eyes & \multicolumn{2}{|c|}{ Number of eyes detected } & \multicolumn{2}{|c}{ Number of false eyes } \\
\hline 336 & 310 & $92.26 \%$ & 31 & $9.09 \%$ \\
\hline
\end{tabular}

To show the quality of our method, we have shown in Table 5, the results of the four selected images from 50 images used to test our method and that are characterized by complex background and contains face the profile. 
Table 5. Steps of Detection by our Method on Complex Images
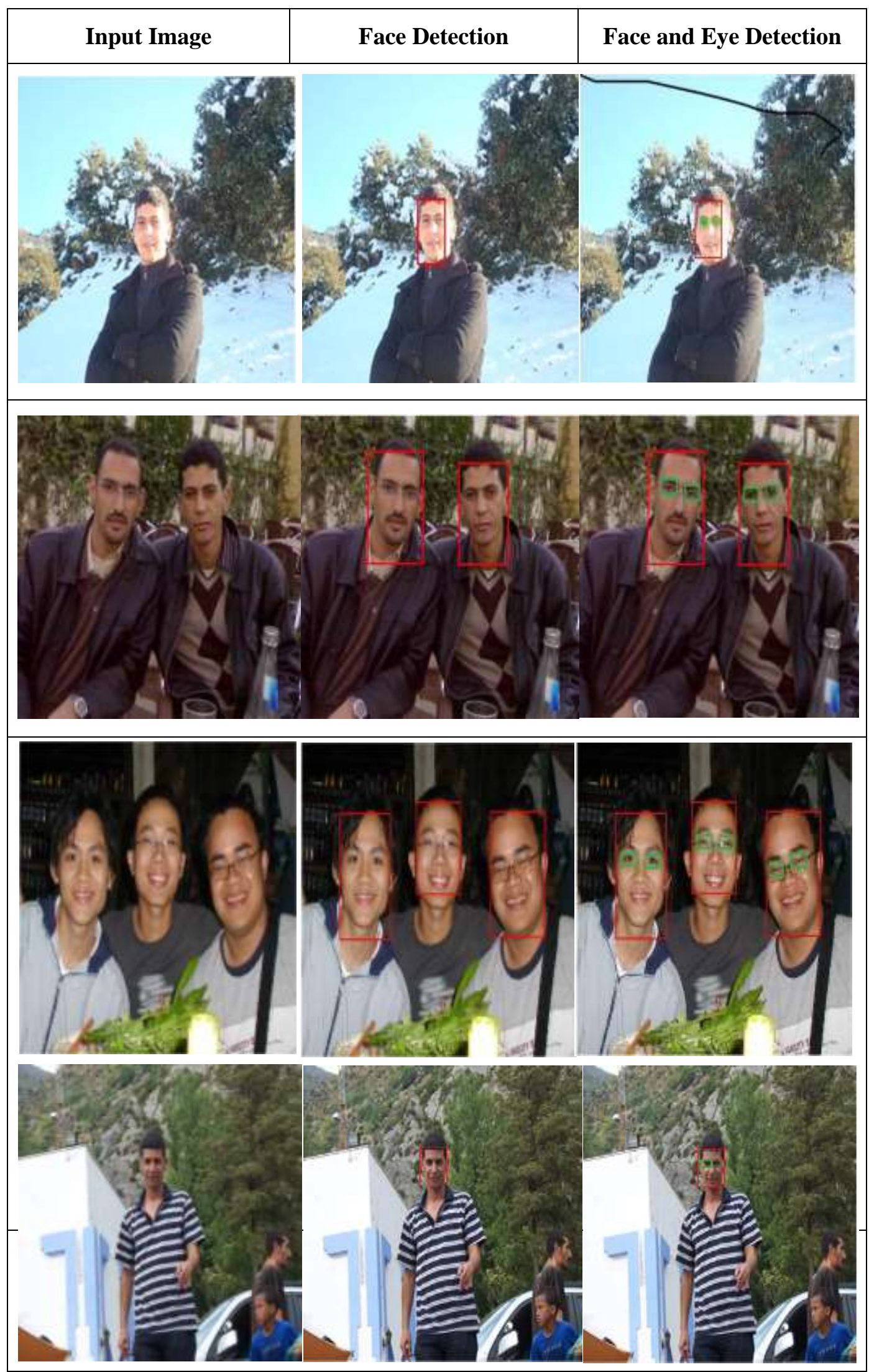


\section{Conclusion}

In this paper, we presented an eye detection method based on the Viola-Jones detector, the skin color and the application of an eye template. Under all conditions of pose, expression or the presence of occultation, our method provides perfect eye detection. The results obtained show that this technique has many advantages in terms of the quality and the speed of the detection.

\section{References}

[1] S.-I. Choi, Y. Lee and C. Kim, "Confidence Measure Using Composite Features for Eye Detection in a Face Recognition System”, IEEE Signal Processing Letters, vol. 22, no. 2, (2015), pp. 225-228.

[2] M. Hassaballah and S. Aly, "Face recognition: challenges, achievements and future directions", IET Computer Vision, vol. 9, no. 4, (2015), pp. 614-626.

[3] Q. Liu, W. Zhang, H. Li and K. Ngi Ngan, "Hybrid Human Detection and Recognition in Surveillance", Elsevier, Neurocomputing, vol. 194, (2016), pp. 10-23.

[4] C. Rougier, J. Meunier, A. St-Arnaud and J. Rousseau, "Robust Video Surveillance for Fall Detection Based on Human Shape Deformation", IEEE Transactions on Circuits and Systems for Video Technology, vol. 21, no. 5, (2011), pp. 611-622.

[5] M. Hameed Siddiqi, R. Ali, A. Mehmood Khan, E. Soo Kim, G. Junghyun Kim and S. Lee, "Facial expression recognition using active contour-based face detection, facial movement-based feature extraction, and non-linear feature selection", Multimedia Systems, vol. 21, (2015), pp.541-555.

[6] Y. Muttu and H. G. Virani, "Effective Face Detection, Feature Extraction \& Neural Network Based Approaches for Facial Expression Recognition", International Conference on Information Processing (ICIP) Vishwakarma Institute of Technology, (2015), pp. 102-107.

[7] Y. Fu, G. Guo and T. S. Huang, "Age Synthesis and Estimation via Faces: A Survey”, IEEE Transactions On Pattern Analysis and Machine Intelligence, vol. 32, no. 11, (2010), pp. 1955-1976.

[8] P. M. Corcoran, F. Nanu, S. Petrescu and P. Bigioi, "Real-Time Eye Gaze Tracking for Gaming Design and Consumer Electronics Systems", IEEE Transactions on Consumer Electronics, vol. 58, no. 2, (2012), pp. 347-355.

[9] Y. Ebisawa and K. Fukumoto, "Head-Free, Remote Eye-Gaze Detection System Based on Pupil-Corneal Reflection Method with Easy Calibration Using Two Stereo-Calibrated Video Cameras", IEEE Transactions On Biomedical Engineering, vol. 60, no. 10, (2013), pp. 2952-2960.

[10] B. Cyganek and S. Gruszczyński, "Hybrid computer vision system for drivers' eye recognition and fatigue monitoring", Elsevier, Neurocomputing, vol. 126, (2014), pp. 78-94.

[11] L. Fattouh Ibrahim, M. Abulkhair, A. D. AlShomrani, M. ALGarni, A. AL-Mutiry, F. AL-Gamdi and R. Kalenen, "Using Haar classifiers to detect driver fatigue and provide alertse", Multimed Tools Appl, vol. 71, (2014), pp. 1857-1877.

[12] T.-H. Chang and Y.-R. Chen, "Driver Fatigue Surveillance via Eye Detection", IEEE 17th International Conference on Intelligent Transportation Systems (ITSC), Qingdao, China, (2014), pp. 366-371.

[13] D. Gonzalez-Ortega, F. J. Diaz-Pernas, M. Anton-Rodriguez, M. MartinezZarzuela and J. F. Diez-Higuera, "Real-time vision-based eye state detection for driver alertness monitoring", Pattern Anal Applic, vol. 16, (2013), pp. 285-306.

[14] F. Song, X. Tan, S. Chen and Z.-H. Zhou, "A literature survey on robust and efficient eye localization in real-life scenarios", Elsevier, Pattern Recognition, vol. 46, no. 12, (2013), pp. 3157-3173.

[15] D. Witzner Hansen and Q. Ji, "In the Eye of the Beholder: A Survey of Models for Eyes and Gaze", IEEE Transactions On Pattern Analysis and Machine Intelligence, vol. 32, no. 3, (2010), pp. 478-500.

[16] K. Nanaa, M. Rizon, M. Nordin Abd Rahman, A. Almejrad, A. Zaliha Abd Aziz and S. Bahri Mohamed, "Eye Detection Using Composite Cross-Correlation", American Journal of Applied Sciences, vol. 10, (2013), pp. 1448-1456.

[17] R. F. Abdel-Kader, R. Atta and S. El-Shakhabe, "An efficient eye detection and tracking system based on particle swarm optimization and adaptive block-matching search algorithm", Elsevier, Engineering Applications of Artificial Intelligence, vol. 31, (2014), pp. 90-100.

[18] J. Songa, Z. Chia and J. Liu, "A Robust Eye Detection Method Using Combined Binary Edge and Intensity Information”, Elsevier, Pattern Recognition, vol. 39, (2006), pp. 1110-1125.

[19] L. Kumar Bhatta and D. Rana, "Facial Feature Extraction of Color Image using Gray Scale Intensity Value”, International Journal of Engineering Research \& Technology (IJERT), vol. 3, no. 3, (2014), pp. 1177-1180.

[20] M. Affan Zia, U. Ansari, M. Jamil, O. Gillani and Y. Ayaz, "Face and Eye Detection in Images using Skin Color Segmentation and Circular Hough Transform", 2014 International Conference on Robotics and Emerging Allied Technologies in Engineering (iCREATE), Islamabad, Pakistan, (2014), pp. 211-213.

[21] D. Monzo, A. Albiol, J. Sastre and A. Albiol, "Precise eye localization using HOG descriptors", Machine Vision and Applications, vol. 22, (2011), pp. 471-480. 
[22] R. Sharma and A. Savakis, "Lean histogram of oriented gradients features for effective eye detection", Journal of Electronic Imaging, vol. 24, no. 6, (2015), pp. 1-12.

[23] P. Sudhakara Rao Vijayalaxmi and S. Sreehari, "Neural Network Approach for Eye Detection", Computer Science \& Information Technology (CS \& IT), vol. 2, (2012), pp. 269-281.

[24] K. Rusek and P. Guzik, "Two-stage neural network regression of eye location in face images", Multimedia Tools and Applications (Open Access), (2014), pp. 1-14

[25] P. Wang and Q. Ji, "Multi-View Face Detection under Complex Scene based on Combined SVMs", Proceeding IEEE, Int'l. Conf. on Pattern Recognition, (2004).

[26] A. Cheddad, D. Mohamad and A. Abd Manaf, "Exploiting Voronoi diagram properties in face segmentation and feature extraction", Elsevier, Pattern Recognition, vol. 141, (2008), pp. 3842-3859.

[27] M. Jian, K.-M. Lam and J. Dong, "Facial-feature detection and localization based on a hierarchical scheme", Elsevier, Information Sciences, vol. 262, (2014), pp. 1-14.

[28] S. El Kaddouhi, A. Saaidi and M. Abarkan, "A New Robust Face Detection Method Based on Corner Points", International Journal of Software Engineering and Its Applications, vol. 8, no. 11, (2014), pp. 2540.

[29] M. Yu, Y. Lin and X. Wang, "An efficient hybrid eye detection method", Turkish Journal of Electrical Engineering \& Computer Sciences, vol. 24, (2016), pp. 1586-1603.

[30] F. Y. Shih, S. Cheng and C.-F. Chuang, "Extracting Faces and Facial Features from Color Images", International Journal of Pattern Recognition and Artificial Intelligence, vol. 22, no. 3, (2008), pp. 515534.

[31] M. Hassaballah, T. Kanazawa and S. Ido, "Efficient eye detection method based on grey intensity variance and independent components analysis", IET Computer Vision, vol. 4, no. 4, (2010), pp. 261-271.

[32] Z. Han, T. Su, Z. Ou and W. Xu, "Precise localization of eye centers with multiple cues", Multimedia Tools and Applications, vol. 68, no. 3, (2014), pp. 931-945.

[33] S. El Kaddouhi, A. Saaidi, and M. Abarkan, "Eye detection based on the Viola-Jones method and corner points", Multimedia Tools and Applications, vol. 76, (2017), pp. 23077-23097.

[34] C. Jung, T. Sun and L. Jiao, "Eye detection under varying illumination using the retinex theory", Elsevier, Neurocomputing, vol. 113, (2013), pp. 130-137.

[35] P. Viola and M. Jones, "Robust real-time face detection", International Journal of Computer Vis, vol.57, no.2, (2004), pp.137-154.

[36] S. Lam Phung, A. Bouzerdoum and D. Chai, "Skin Segmentation Using Color Pixel Classification: Analysis and Comparison”, IEEE Transactions on Pattern Analysis and Machine Intelligence, vol. 27, no. 1, (2005), pp. 148-154.

[37] The FEI face database. http://fei.edu.br/ cet/facedatabase.html, (2014).

\section{Authors}

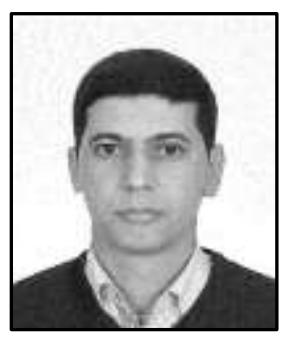

Samir El Kaddouhi, he received the master's degrees from Faculty of Sciences, Tetouan in 2010. He is currently working toward the PhD degree in the Laboratory of Engineering science (LSI) at the Faculty Polydisciplinaire of Taza. His current research interests include computer vision, artificial intelligence, optimization, face recognition, face detection.

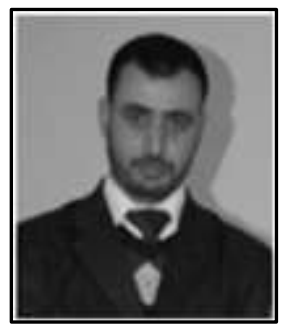

Abderrahim Saaidi, he received the PhD degree from SMBAFez University in 2010. He is currently a professor of computer science at SMBA-Taza University. He is member of the LIIAN and LSI Laboratories. His research interests include camera selfcalibration, 3D reconstruction and real-time rendering. 


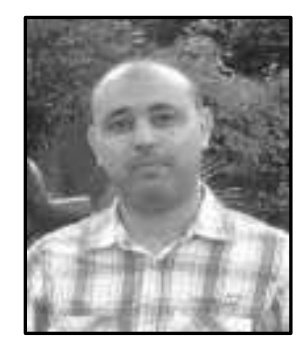

Mustapha Abarkan, he received the DEA and PhD degrees in optoelectronic from the University of Metz, in France, in 1998 and 2002 , respectively. His research was devoted to the study of nonlinear optical crystals for electro-optic Q-switching of laser resonators. He then performed thesis works at LMOPS (laboratory Optical Materials, Photonic and Systems, Metz, France). He became Assistant Professor at the University of Fès, in Morocco in 2003 where he received the HDR professor in 2007. Since 2012, he leads researches and works in computer vision. He is member of the Laboratory of Engineering science (LSI) at the Faculty Polydisciplinaire of Taza, in Marocco. 